\title{
An Iteratively Reweighted Least Squares Algorithm for Sparse Regularization
}

\author{
Sergey Voronin and Ingrid Daubechies
}

\begin{abstract}
We present a new algorithm and the corresponding convergence analysis for the regularization of linear inverse problems with sparsity constraints, applied to a new generalized sparsity promoting functional. The algorithm is based on the idea of iteratively reweighted least squares, reducing the minimization at every iteration step to that of a functional including only $\ell_{2}$-norms. This amounts to smoothing of the absolute value function that appears in the generalized sparsity promoting penalty we consider, with the smoothing becoming iteratively less pronounced. We demonstrate that the sequence of iterates of our algorithm converges to a limit that minimizes the original functional.
\end{abstract}

\section{Introduction}

Over the last several years, an abundant number of algorithms (e.g. [4, 2, 17, 16]) have been proposed for the minimization of the $\ell_{1}$-penalized functional $F_{\tau}(x)=\|A x-b\|_{2}^{2}+2 \tau\|x\|_{1}$, where the matrix $A$, the vector $x$ and the constant $\tau$ are, respectively, in $\mathbb{R}^{M \times N}, \mathbb{R}^{N}$ and $\mathbb{R}_{+}$. This functional has a number of interesting applications, such as image restoration [6], face recognition [15], and in inverse problems from geophysics [10]; one can also view the recovery of corrupted low rank matrices as a generalization (since it typically penalizes the $\ell_{1}$ norm of the singular values, i.e. the nuclear norm of the matrix) [14]. The $\|x\|_{1}=\sum_{k=1}^{N}\left|x_{k}\right|$ penalty is the closest norm to the $\ell_{0}$-penalty (the count of non-zeros in a signal), and the relationship between the two has been brought into focus by compressive sensing [3]. Since $\|x\|_{1}$ is not differentiable due to the absolute value function $|\cdot|$, standard gradient based techniques cannot be directly applied for the minimization of $F_{\tau}$. In this paper, we consider a more general functional of which $F_{\tau}$ is a particular case. The new functional introduced in [11] which the algorithm in this paper can minimize is $F_{\mathbf{q}, \boldsymbol{\lambda}}(x)$ :

$$
F_{\mathbf{q}, \boldsymbol{\lambda}}(x)=\|A x-b\|_{2}^{2}+2 \sum_{k=1}^{N} \lambda_{k}\left|x_{k}\right|^{q_{k}}
$$

where the coefficients $q_{k}$ and $\lambda_{k}$ may be different for each $1 \leq k \leq N$, with $1 \leq q_{k} \leq 2$ for each $k$. The more general functional makes it possible to treat different components of $x$ differently, corresponding to their different roles. A simple example with a half sparse, half dense signal is illustrated in the Numerics section; in that case, imposing a sparsity inducing penalty on all coefficients is not ideal for proper recovery. Another important instance is the case when the penalization contains a multiscale representation (e.g. the wavelet decomposition) of an object to be reconstructed/approximated. In this case, one has an extra matrix $W$, representing the transform to wavelet coefficients, and the minimization problem for $w=W x$ takes the form:

$$
\bar{w}=\arg \min _{w}\left\{\left\|A W^{-1} w-b\right\|_{2}^{2}+\sum_{k=1}^{N} \lambda_{k}\left|w_{k}\right|^{q_{k}}\right\}
$$


If $W$ is a wavelet transform, then the different entries of the vector $w$ fulfill distinctly different roles, with some being responsible for coarse scales and others for fine details. In this case, the total number of possible coefficients corresponding to coarse scales is typically quite limited, with each of them crucial to the overall model (e.g. [10]). Thus, we do not necessarily want to impose a sparsity-promoting penalty on these coefficients, which means we would choose $q_{k}>1$ for them in the penalty function. On the other hand, the coefficients corresponding to fine scales are typically quite sparse in the object to be reconstructed, and the inversion procedure might, without appropriate regularization, be prone to populate them with noisy features; in this case, a sparsity promoting choice $q_{k}=1$ would be indicated for those $k$.

Two approaches are commonly used by various algorithms for the minimization of functionals that, like $F_{\mathbf{q}, \boldsymbol{\lambda}}$, involve a non-smooth absolute value term. The first approach handles the non-smooth minimization problem directly. For instance, for our original $F_{\tau}$, one uses the soft-thresholding operation [4] on $\mathbb{R}$, defined by:

$$
S_{\tau}(x)= \begin{cases}x-\tau, & x \geq \tau \\ 0, & -\tau \leq x \leq \tau ; \\ x+\tau, & x \leq-\tau .\end{cases}
$$

For a vector of $N$ elements, soft-thresholding is then defined component-wise by setting, $\forall k=1, \ldots, N$, $\left(\mathbb{S}_{\tau}(x)\right)_{k}=S_{\tau}\left(x_{k}\right)$. The use of soft-thresholding relies on the identity $S_{\tau}(\beta)=\arg \min _{a}\left\{(a-\beta)^{2}+2 \tau|a|\right\}$ for scalars $a$ and $\beta$, which for vectors $x$ and $b$ translates to:

$$
\mathbb{S}_{\tau}(b)=\arg \min _{x}\left\{\|x-b\|_{2}^{2}+2 \tau\|x\|_{1}\right\}
$$

The simplest example is the Iterative Soft Thresholding Algorithm (ISTA) [4]:

$$
x^{n+1}=\mathbb{S}_{\tau}\left(x^{n}+A^{T} b-A^{T} A x^{n}\right)
$$

which for an initial $x^{0}$ and with $\|A\|_{2}<1$ (easily accomplished by rescaling; $\|A\|_{2}$ is the operator norm of $A$ from $\ell_{2}$ to $\ell_{2}$, also called the spectral norm of $A$ ), converges slowly but surely to the $\ell_{1}$-minimizer. A faster variation on this scheme, known as FISTA [1], is frequently employed; the thresholding function can also be adjusted to correspond to more general penalties [12]. Along the same line of thinking, algorithms based on the dual space of the $\ell_{1}$-norm have been proposed [16], with the dual being the $\ell_{\infty}$-norm.

The second approach to algorithms minimizing the $\ell_{1}$-based functional involves some kind of smoothing. One idea is to replace the entire functional by a smooth approximation. This can be done, for instance, by convolving the absolute value function with narrow Gaussians [13]. This approach then allows for the use of standard gradient based methods (such as Conjugate Gradients) for the minimization of the approximate smooth functional. The main problem with this approach is that we are then minimizing a slightly different functional from the original that does not necessarily have the same properties that the original penalty possesses.

The algorithm described in this paper replaces the $\left|x_{k}\right|^{q_{k}}$ term in $F_{\mathbf{q}, \boldsymbol{\lambda}}$ with a smoothened version that tends to the original as the iterates progress towards the limit. This algorithm builds upon the original iteratively reweighted least squares (or IRLS) method proposed in [5] (as well as earlier work in [6, 8]), extending it to the unconstrained case and to a more general penalty. The idea can be illustrated simplest for the $q_{k}=1$ case. Consider the approximation:

$$
\left|x_{k}\right|=\frac{x_{k}^{2}}{\left|x_{k}\right|}=\frac{x_{k}^{2}}{\sqrt{x_{k}^{2}}} \approx \frac{x_{k}^{2}}{\sqrt{x_{k}^{2}+\epsilon^{2}}}
$$

where in the rightmost term, a small $\epsilon \neq 0$ is used, to insure the denominator is finite, regardless of the value of $x_{k}$. Thus, at the $n$-th iteration, a reweighted $\ell_{2}$-approximation to the $\ell_{1}$-norm of $x$ is of the form:

$$
\|x\|_{1} \approx \sum_{k=1}^{N} \frac{x_{k}^{2}}{\sqrt{\left(x_{k}^{n}\right)^{2}+\epsilon_{n}^{2}}}=\sum_{k=1}^{N} \tilde{w}_{k}^{n} x_{k}^{2}
$$


where the right hand side is a reweighted two-norm with weights:

$$
\tilde{w}_{k}^{n}=\frac{1}{\sqrt{\left(x_{k}^{n}\right)^{2}+\epsilon_{n}^{2}}} .
$$

Clearly, it follows that $\sum_{k} \tilde{w}_{k}^{n}\left(x_{k}^{n}\right)^{2}$ is a close approximation to $\left\|x^{n}\right\|_{1}$. In the same way, we can use the slightly more general weights:

$$
w_{k}^{n}=\frac{1}{\left[\left(x_{k}^{n}\right)^{2}+\epsilon_{n}^{2}\right]^{\frac{2-q_{k}}{2}}} .
$$

for the approximation $\left|x_{k}^{n}\right|^{q_{k}} \approx w_{k}^{n}\left(x_{k}^{n}\right)^{2}$ to hold; these can then deal with the case $1 \leq q_{k} \leq 2$.

We shall use a sequence $\left\{\epsilon_{n}\right\}$ such that $\epsilon_{n} \rightarrow 0$ as $n \rightarrow \infty$. We note that it is important that $\epsilon_{n}>0$ for all $n$ for a rigorous convergence proof. In an approach where $\epsilon_{n}=0$, entries $k$ for which $x_{k}^{n}=0$ would lead to diverging $\tilde{w}_{k}^{n}$ and hence to $x_{k}^{n^{\prime}}=0$ for all subsequent $n^{\prime}>n$. This is OK if the $k$-th entry of the minimizer is indeed zero; if (as is typically the case) this cannot be guaranteed, convergence would fail.

A precise choice of the sequence $\left\{\epsilon_{n}\right\}$ is important for convergence analysis. Although in practice, different approaches can work, the rate at which the $\epsilon$-sequence converges needs to match that of the iterates $x^{n}$. In our analysis, we will use the following definition:

$$
\epsilon_{n}=\min \left(\epsilon_{n-1},\left(\left\|x^{n}-x^{n-1}\right\|_{2}+\alpha^{n}\right)^{\frac{1}{2}}\right),
$$

where $\alpha \in(0,1)$ is some fixed number. The resulting algorithm we present and analyze is very similar in form to $(1.2)$ :

$$
x_{k}^{n+1}=\frac{1}{1+\lambda_{k} q_{k} w_{k}^{n}}\left(x_{k}^{n}+\left(A^{T} b\right)_{k}-\left(A^{T} A x^{n}\right)_{k}\right) \quad \text { for } \quad k=1, \ldots, N,
$$

with the thresholding replaced by an iteration-dependent scaling operation using the weights (1.4). The algorithm is found to be numerically competitive with the thresholding based schemes for the $\ell_{1}$-case but has the advantage that it can handle the minimization of more general functionals of the form $F_{\mathbf{q}, \boldsymbol{\lambda}}$. The main contribution of this paper is a detailed proof of convergence, the methodology of which can be readily applied to analyze similar schemes. An added advantage of a scheme in which all terms are quadratic in the unknown $x$ is that it can be combined with a conjugate gradient approach to speed up the algorithm. In [11] such an algorithm was proposed, and convergence proved if at each reweighted step, the conjugate gradient scheme was pursued to convergence. In [7], the more general and more realistic situation is considered, where only some conjugate gradient steps are taken at each iteration. In both cases, the choice of $\left\{\epsilon_{n}\right\}$ (e.g. (1.5)), remains crucial for the convergence analysis.

\section{Constructions}

2.1. Analysis of the generalized sparsity inducing functional. Here, we derive and comment on the optimality conditions of the functional:

$$
F(x)=\|A x-b\|_{2}^{2}+2 \sum_{k=1}^{N} \lambda_{k}\left|x_{k}\right|^{q_{k}},
$$

for the range $1 \leq q_{k} \leq 2$, where in (2.1), we drop the subscripts $\mathbf{q}$ and $\boldsymbol{\lambda}$ for convenience. Notice that since (2.1) is convex for the range of $q_{k}$ specified, every local minimizer is a global minimizer of the functional. The optimality conditions for a general vector $x$ with components $x_{k}$ for $k \in(1, \ldots, N)$ can be written down in component-wise form, as derived in Lemma 2.1 below. Note that as $F_{\mathbf{1}, \lambda}$ is a special case of $F_{\mathbf{q}, \boldsymbol{\lambda}}$, the component-wise conditions below reduce to the well known optimality conditions of the $\ell_{1}$ penalized functional when $q_{k}=1$ for all $k$. 
LEMMA 2.1. The conditions for the minimizer of the functional $F(x)$ as defined in (2.1) are:

$$
\begin{aligned}
& \left\{A^{T}(b-A x)\right\}_{k}=\lambda_{k} \operatorname{sgn}\left(x_{k}\right) q_{k}\left|x_{k}\right|^{q_{k}-1}, \quad x_{k} \neq 0 \quad\left(1 \leq q_{k} \leq 2\right) \\
& \left\{A^{T}(b-A x)\right\}_{k}=0, \quad x_{k}=0 \quad\left(q_{k}>1\right) \\
& \left|\left\{A^{T}(b-A x)\right\}_{k}\right| \leq \lambda_{k}, \quad x_{k}=0 \quad\left(q_{k}=1\right)
\end{aligned}
$$

Proof. Since for the case $1 \leq q_{k} \leq 2, F(x)$ is convex, any local minimizer is necessarily global. Thus, to characterize the minimizer, it is necessary only to work out the conditions corresponding to $F(x) \leq F(x+t z)$ for all sufficiently small $t \in \mathbb{R}$ and all $z \in \mathbb{R}^{N} . F(x) \leq F(x+t z)$ implies that:

$$
t^{2}\|A z\|^{2}+2 t\left\langle z, A^{T}(A x-b)\right\rangle+2 \sum_{k=1}^{N} \lambda_{k}\left(\left|x_{k}+t z_{k}\right|^{q_{k}}-\left|x_{k}\right|^{q_{k}}\right) \geq 0 .
$$

We derive $N$ conditions, one for each index $k \in\{1, \ldots, N\}$; for the $k$-th condition, we consider $z$ of the special form $z=z_{k} e_{k}$ (i.e. only the $k$-th entry of $z$ differs from 0 ). We separately analyze the cases $x_{k} \neq 0$ and $x_{k}=0$, starting with the former.

When $x_{k} \neq 0$, the function $f(t)=\left|x_{k}+t z_{k}\right|^{q_{k}}$ is $C^{\infty}$ at $t=0$. Using a Taylor series expansion around 0 , we then get $f(t)=f(0)+t f^{\prime}(0)+O\left(t^{2}\right)$. In addition, $\operatorname{sgn}\left(x_{k}+t z_{k}\right)=\operatorname{sgn}\left(x_{k}\right)$ for sufficiently small $t$. Keeping $t$ fixed we analyze both signs of $x_{k}$. For $x_{k}>0$, we have $\operatorname{sgn}\left(x_{k}\right)=1$ and $\left|x_{k}+t z_{k}\right|=x_{k}+t z_{k}$, so that:

$$
f(t)=\left(x_{k}+t z_{k}\right)^{q_{k}} \Longrightarrow f^{\prime}(t)=\operatorname{sgn}\left(x_{k}\right) q_{k} z_{k}\left(x_{k}+t z_{k}\right)^{q_{k}-1}=\operatorname{sgn}\left(x_{k}\right) q_{k} z_{k}\left|x_{k}+t z_{k}\right|^{q_{k}-1} .
$$

When $x_{k}<0$, we have $\operatorname{sgn}\left(x_{k}\right)=-1$ and $\left|x_{k}+t z_{k}\right|=-\left(x_{k}+t z_{k}\right)$, so that:

$$
f(t)=\left(-x_{k}-t z_{k}\right)^{q_{k}} \Longrightarrow f^{\prime}(t)=-q_{k} z_{k}\left(-x_{k}-t z_{k}\right)^{q_{k}-1}=\operatorname{sgn}\left(x_{k}\right) q_{k} z_{k}\left|x_{k}+t z_{k}\right|^{q_{k}-1} .
$$

Thus, $f^{\prime}(0)=\operatorname{sgn}\left(x_{k}\right) q_{k} z_{k}\left|x_{k}\right|^{q_{k}-1}$ for all $x_{k} \neq 0$. Thus, there exists a constant $C>0$ such that the Taylor expansion of $f$ becomes:

$$
\begin{aligned}
f(t) & =\left|x_{k}+t z_{k}\right|^{q_{k}}=\left|x_{k}\right|^{q_{k}}+t \operatorname{sgn}\left(x_{k}\right) q_{k} z_{k}\left|x_{k}\right|^{q_{k}-1}+O\left(t^{2}\right) \\
& \leq\left|x_{k}\right|^{q_{k}}+t \operatorname{sgn}\left(x_{k}\right) q_{k} z_{k}\left|x_{k}\right|^{q_{k}-1}+C t^{2},
\end{aligned}
$$

This implies in particular that $\left|x_{k}+t z_{k}\right|^{q_{k}}-\left|x_{k}\right|^{q_{k}} \leq t \operatorname{sgn}\left(x_{k}\right) q_{k} z_{k}\left|x_{k}\right|^{q_{k}-1}+C t^{2}$. Using this and $z=z_{k} e_{k}$ in (2.3) gives:

$$
\begin{aligned}
& t^{2}\left\|A\left(z_{k} e_{k}\right)\right\|^{2}+2 t\left\langle z_{k} e_{k}, A^{T}(A x-b)\right\rangle+2 \lambda_{k}\left(t \operatorname{sgn}\left(x_{k}\right) q_{k} z_{k}\left|x_{k}\right|^{q_{k}-1}+C t^{2}\right) \geq 0 \\
\Longrightarrow & t^{2}\left(\left\|A\left(z_{k} e_{k}\right)\right\|^{2}+2 C \lambda_{k}\right)+2 t\left(z_{k}\left\{A^{T}(A x-b)\right\}_{k}+\lambda_{k} \operatorname{sgn}\left(x_{k}\right) q_{k} z_{k}\left|x_{k}\right|^{q_{k}-1}\right) \geq 0 .
\end{aligned}
$$

The first term can be made arbitrary small with respect to the second; the inequality will thus hold for both $t>0$ and $t<0$ iff:

which leads to:

$$
z_{k}\left\{A^{T}(A x-b)\right\}_{k}+\lambda_{k} \operatorname{sgn}\left(x_{k}\right) q_{k} z_{k}\left|x_{k}\right|^{q_{k}-1}=0
$$

$$
\left\{A^{T}(b-A x)\right\}_{k}=\lambda_{k} \operatorname{sgn}\left(x_{k}\right) q_{k}\left|x_{k}\right|^{q_{k}-1}, x_{k} \neq 0 .
$$

Note that when $q_{k}=1$ we recover the familiar condition for minimization of the $\ell_{1}$-functional:

$$
\left\{A^{T}(b-A x)\right\}_{k}=\lambda_{k} \operatorname{sgn}\left(x_{k}\right), x_{k} \neq 0 .
$$

When $x_{k}=0$, recalling that $z=z_{k} e_{k},(2.3)$ gives:

$$
t^{2}\left\|A\left(z_{k} e_{k}\right)\right\|^{2}+2 t\left\langle z_{k} e_{k}, A^{T}(A x-b)\right\rangle+2 \lambda_{k}|t|^{q_{k}}\left|z_{k}\right|^{q_{k}} \geq 0 .
$$

Making the substitutions $t^{2}=|t|^{2}, t=|t| \operatorname{sgn}(t)$, we obtain

$$
|t|^{2} \|\left. A\left(z_{k} e_{k}\right)\right|^{2}+|t|\left(2 \operatorname{sgn}(t) z_{k}\left\{A^{T}(A x-b)\right\}_{k}+2 \lambda_{k}|t|^{q_{k}-1}\left|z_{k}\right|^{q_{k}}\right) \geq 0 .
$$

In this case, we have to consider the case $q_{k}=1$ and $q_{k}>1$ separately. When $q_{k}>1$ we have that:

$$
|t|^{2}|| A z||^{2}+2 \lambda_{k}|t|^{q_{k}}\left|z_{k}\right|^{q_{k}}+2|t| \operatorname{sgn}(t) z_{k}\left\{A^{T}(A x-b)\right\}_{k} \geq 0 \text {. }
$$


Since $q_{k}>1$, the first two terms on the left have greater powers of $|t|$ than the last term and can be made arbitrarily smaller by picking $t$ small enough. This means we must have:

$$
2 \operatorname{sgn}(t) z_{k}\left\{A^{T}(A x-b)\right\}_{k} \geq 0
$$

for all $t$, which can be true only if $\left\{A^{T}(A x-b)\right\}_{k}=0$. Thus, we conclude that the condition is:

$$
\left\{A^{T}(b-A x)\right\}_{k}=0, x_{k}=0 \quad\left(q_{k}>1\right) .
$$

For $q_{k}=1$, applying a similar argument to (2.5) leads to:

$$
\begin{aligned}
& |t|^{2}|| A z\left|\|^{2}+\right| t \mid\left(2 \operatorname{sgn}(t) z_{k}\left\{A^{T}(A x-b)\right\}_{k}+2 \lambda_{k}\left|z_{k}\right|\right) & \geq 0 \\
& \operatorname{sgn}(t) z_{k}\left\{A^{T}(A x-b)\right\}_{k}+\lambda_{k}\left|z_{k}\right| & \geq 0 .
\end{aligned}
$$

Now consider the two cases: where $t$ has the same sign as $z_{k}, \operatorname{sgn}(t)=\operatorname{sgn}\left(z_{k}\right)$ or the opposite $\operatorname{sign}, \operatorname{sgn}(t)=$ $-\operatorname{sgn}\left(z_{k}\right)$. They lead to, respectively:

$$
\left\{A^{T}(A x-b)\right\}_{k}+\lambda_{k} \geq 0 \text { and }-\left\{A^{T}(A x-b)\right\}_{k}+\lambda_{k} \geq 0,
$$

so we obtain the condition:

$$
\left|\left\{A^{T}(b-A x)\right\}_{k}\right| \leq \lambda_{k}, x_{k}=0 \quad\left(q_{k}=1\right) .
$$

Thus, we can summarize the component-wise conditions for the minimizer of $F(x)$ as in (2.2).

The conditions derived in Lemma 2.1 allow us to pick a strategy for selecting $\left\{\lambda_{k}\right\}$. As an example, for the case $q_{k}=1$ and $\lambda_{k}=\lambda$ for all $k$ we have that for $\lambda>\left\|A^{T} b\right\|_{\infty}$, the optimal solution is the zero vector. Hence, we typically would start at some value of $\lambda$ just below $\left\|A^{T} b\right\|_{\infty}$ where the zero vector is a good initial guess. We can then iteratively decrease $\lambda$ and use the previous solution as the initial guess at the next lower $\lambda$ while we go down to some target residual. Well-known techniques such as the L-curve method [9] apply here.

2.2. Derivation of the algorithm. The iteratively reweighted least squares (IRLS) algorithm given by scheme (1.6) with weights (1.4) follows from the construction of a surrogate functional (2.6) which we will use in our analysis, as presented in Lemma 2.2 below. In our constructions, we split the index set $1 \leq k \leq N$ into two parts: $Q_{1}=\left\{k: 1 \leq q_{k}<2\right\}$ and $Q_{2}=\left\{k: q_{k}=2\right\}$.

Lemma 2.2. Define the surrogate functional:

$$
\begin{aligned}
G(x, a, w, \epsilon) & =\|A x-b\|_{2}^{2}-\|A(x-a)\|_{2}^{2}+\|x-a\|_{2}^{2} \\
& +\sum_{k \in Q_{1}} \lambda_{k}\left(q_{k} w_{k}\left(\left(x_{k}\right)^{2}+\epsilon^{2}\right)+\left(2-q_{k}\right)\left(w_{k}\right)^{\frac{q_{k}}{q_{k}-2}}\right) \\
& +\sum_{k \in Q_{2}}\left[2 \lambda_{k}\left(\left(x_{k}\right)^{2}+\epsilon^{2}\right)\left(w_{k}^{2}-2 w_{k}+2\right)\right] .
\end{aligned}
$$

Then the minimization procedure $w^{n}=\arg \min _{w} G\left(x^{n}, a, w, \epsilon_{n}\right)$ defines the iteration dependent weights:

$$
w_{k}^{n}=\frac{1}{\left[\left(x_{k}^{n}\right)^{2}+\left(\epsilon_{n}\right)^{2}\right]^{\frac{2-q_{k}}{2}}} .
$$

In addition, the minimization procedure $x^{n+1}=\arg \min _{x} G\left(x, x^{n}, w^{n}, \epsilon_{n}\right)$ produces the iterative scheme:

$$
x_{k}^{n+1}=\frac{1}{1+\lambda_{k} q_{k} w_{k}^{n}}\left(\left(x^{n}\right)_{k}-\left(A^{T} A x^{n}\right)_{k}+\left(A^{T} b\right)_{k}\right) .
$$


Proof. For the derivation of the weights from $w^{n}=\arg \min _{w} G\left(x^{n}, a, w, \epsilon_{n}\right)$, we take only the terms of $G$ that depend on $w$. We derive separately the weights for $k \in Q_{1}$ and $k \in Q_{2}$. First, for $k \in Q_{1}$ :

$$
\begin{aligned}
& \frac{\partial}{\partial w_{k}}\left[q_{k} w_{k}\left(\left(x_{k}^{n}\right)^{2}+\left(\epsilon_{n}\right)^{2}\right)+\left(2-q_{k}\right)\left(w_{k}\right)^{\frac{q_{k}}{q_{k}-2}}\right]=0 \\
\Longrightarrow & q_{k}\left(\left(x_{k}^{n}\right)^{2}+\left(\epsilon_{n}\right)^{2}\right)+\left(2-q_{k}\right) \frac{q_{k}}{q_{k}-2}\left(w_{k}\right)^{\frac{q_{k}}{q_{k}-2}-1}=0 \\
\Longrightarrow & w_{k}^{n}=\frac{1}{\left[\left(x_{k}^{n}\right)^{2}+\left(\epsilon_{n}\right)^{2}\right]^{\frac{2-q_{k}}{2}}} .
\end{aligned}
$$

Next, for $k \in Q_{2}$, we have:

$$
\begin{aligned}
& \frac{\partial}{\partial w_{k}}\left[2 \lambda_{k}\left(\left(x_{k}^{n}\right)^{2}+\left(\epsilon_{n}\right)^{2}\right)\left(w_{k}^{2}-2 w_{k}+2\right)\right]=2 \lambda_{k}\left(\left(x_{k}^{n}\right)^{2}+\left(\epsilon_{n}\right)^{2}\right)\left(2 w_{k}-2\right)=0 \\
& \Longrightarrow w_{k}^{n}=1
\end{aligned}
$$

Notice that this implies that (2.7) is valid for $k$ in both sets $Q_{1}$ and $Q_{2}$ since for $k \in Q_{2}, q_{k}=2$ and (2.7) gives $w_{k}^{n}=1$ as required.

Next, we verify that the definition:

$$
x_{k}^{n+1}=\left\{\arg \min _{x} G\left(x, x^{n}, w^{n}, \epsilon_{n}\right)\right\}_{k}
$$

recovers the iterative scheme (2.8). Using that $w_{k}^{n}=1$ for $k \in Q_{2}$, as just derived, we have:

$$
\begin{aligned}
G\left(x, x^{n}, w^{n}, \epsilon_{n}\right)=\| & A x-b\left\|_{2}^{2}-\right\| A\left(x-x^{n}\right)\left\|_{2}^{2}+\right\| x-x^{n} \|_{2}^{2} \\
& +\sum_{k \in Q_{1}} \lambda_{k}\left(q_{k} w_{k}^{n}\left(\left(x_{k}\right)^{2}+\left(\epsilon_{n}\right)^{2}\right)+\left(2-q_{k}\right)\left(w_{k}^{n}\right)^{\frac{q_{k}}{q_{k}-2}}\right) \\
& +\sum_{k \in Q_{2}}\left[2 \lambda_{k}\left(\left(x_{k}\right)^{2}+\left(\epsilon_{n}\right)^{2}\right)\right] .
\end{aligned}
$$

To prove (2.8), we again separately analyze the cases $k \in Q_{1}$ and $k \in Q_{2}$. We differentiate (2.9) with respect to $x$, then take the $k$-th component and set to zero. For $k \in Q_{1}$, removing terms of (2.9) that do not depend on $x$, we get:

$$
\begin{aligned}
& \frac{\partial}{\partial x_{k}}\left(\|A x-b\|_{2}^{2}-\left\|A\left(x-x^{n}\right)\right\|_{2}^{2}+\left\|x-x^{n}\right\|_{2}^{2}+\sum_{k \in Q_{1}} \lambda_{l} q_{l} w_{l}^{n} x_{l}^{2}\right) \\
= & \frac{\partial}{\partial x_{k}}\left(\|x\|_{2}^{2}-2\left(x, x^{n}+A^{T} b-A^{T} A x^{n}\right)+\sum_{k \in Q_{1}} \lambda_{l} q_{l} w_{l}^{n} x_{l}^{2}\right)=0
\end{aligned}
$$

and the result is:

$$
-2\left\{A^{T} b\right\}_{k}+2\left\{A^{T} A x^{n}\right\}_{k}+2 x_{k}-2 x_{k}^{n}+2 \lambda_{k} q_{k} w_{k}^{n} x_{k}=0 .
$$

Then we solve for $x_{k}$ and define $x_{k}^{n+1}$ to be the result:

$$
\begin{aligned}
x_{k}\left(1+\lambda_{k} q_{k} w_{k}^{n}\right) & =x_{k}^{n}+\left\{A^{T} b\right\}_{k}-\left\{A^{T} A x^{n}\right\}_{k} \\
\Longrightarrow \quad x_{k}^{n+1} & =\frac{1}{1+\lambda_{k} q_{k} w_{k}^{n}}\left\{x^{n}+A^{T} b-A^{T} A x^{n}\right\}_{k} .
\end{aligned}
$$


For $k \in Q_{2}, w_{k}^{n}=1$ and we obtain:

$$
\begin{aligned}
& \frac{\partial}{\partial x_{k}}\left(\|x\|_{2}^{2}-2\left(x, x^{n}+A^{T} b-A^{T} A x^{n}\right)+\sum_{k \in Q_{2}} 2 \lambda_{k} x_{k}^{2}\right) \\
& =-2\left\{A^{T} b\right\}_{k}+2\left\{A^{T} A x^{n}\right\}_{k}+2 x_{k}-2 x_{k}^{n}+4 \lambda_{k} x_{k}=0 .
\end{aligned}
$$

which, upon solving for $x_{k}$, yields the scheme:

$$
x_{k}^{n+1}=\frac{1}{1+2 \lambda_{k}}\left\{x^{n}+A^{T} b-A^{T} A x^{n}\right\}_{k} .
$$

Thus, it follows that (2.8) holds for all $1 \leq k \leq N$.

Remark 2.3. Assume that as $n \rightarrow \infty, x^{n} \rightarrow x$ and $\epsilon_{n} \rightarrow 0$. Notice that with the weights in (2.7), we have that:

$$
w_{k}^{n}\left(x_{k}^{n}\right)^{2}=\frac{\left(x_{k}^{n}\right)^{2}}{\left(\left(x_{k}^{n}\right)^{2}+\left(\epsilon_{n}\right)^{2}\right)^{\frac{2-q_{k}}{2}}} \rightarrow \frac{x_{k}^{2}}{\left(x_{k}^{2}+0\right)^{\frac{2-q_{k}}{2}}}=\left|x_{k}\right|^{q_{k}} \quad \text { as } n \rightarrow \infty, \text { if } x_{k} \neq 0 .
$$

Next, observe the result of the computation:

$$
\begin{aligned}
& q_{k} w_{k}^{n}\left(\left(x_{k}\right)^{2}+\left(\epsilon_{n}\right)^{2}\right)+\left(2-q_{k}\right)\left(w_{k}^{n}\right)^{\frac{q_{k}}{q_{k}-2}} \\
= & q_{k}\left(\left(x_{k}^{n}\right)^{2}+\left(\epsilon_{n}\right)^{2}\right)^{\left(\frac{q_{k}-2}{2}+\frac{2}{2}\right)}+\left(2-q_{k}\right)\left(\left(x_{k}^{n}\right)^{2}+\left(\epsilon_{n}\right)^{2}\right)^{\left(\frac{q_{k}-2}{2} \frac{q_{k}}{q_{k}-2}\right)} \\
= & 2\left(\left(x_{k}^{n}\right)^{2}+\left(\epsilon_{n}\right)^{2}\right)^{\frac{q_{k}}{2}} .
\end{aligned}
$$

It follows from (2.9) and $q_{k}=2, w_{k}^{n}=1$ for $k \in Q_{2}$ that:

$$
\begin{aligned}
G\left(x^{n}, x^{n}, w^{n}, \epsilon_{n}\right)=\left\|A x^{n}-b\right\|_{2}^{2} & +\sum_{k \in Q_{1}} \lambda_{k}\left(q_{k} w_{k}^{n}\left(\left(x_{k}\right)^{2}+\left(\epsilon_{n}\right)^{2}\right)+\left(2-q_{k}\right)\left(w_{k}^{n}\right)^{\frac{q_{k}}{q_{k}-2}}\right) \\
& +\sum_{k \in Q_{2}}\left[2 \lambda_{k}\left(\left(x_{k}\right)^{2}+\left(\epsilon_{n}\right)^{2}\right)\right],
\end{aligned}
$$

which using (2.10), reduces to:

$$
\left\|A x^{n}-b\right\|_{2}^{2}+2 \sum_{k \in Q_{1}} \lambda_{k}\left(\left(x_{k}^{n}\right)^{2}+\left(\epsilon_{n}\right)^{2}\right)^{\frac{q_{k}}{2}}+2 \sum_{k \in Q_{2}} \lambda_{k}\left(\left(x_{k}^{n}\right)^{2}+\left(\epsilon_{n}\right)^{2}\right)^{\frac{2}{2}} .
$$

Thus, we recover:

$$
G\left(x^{n}, x^{n}, w^{n}, \epsilon_{n}\right)=\left\|A x^{n}-b\right\|_{2}^{2}+2 \sum_{k=1}^{N} \lambda_{k}\left(\left(x_{k}^{n}\right)^{2}+\left(\epsilon_{n}\right)^{2}\right)^{\frac{q_{k}}{2}},
$$

As $n \rightarrow \infty$, assuming $x^{n} \rightarrow x$ and $\epsilon_{n} \rightarrow 0$, we have that:

$$
\lim _{n \rightarrow \infty} G\left(x^{n}, x^{n}, w^{n}, \epsilon_{n}\right)=\|A x-b\|_{2}^{2}+2 \sum_{k=1}^{N} \lambda_{k}\left|x_{k}\right|^{q_{k}},
$$

so we recover the functional (2.1) we would like to minimize. 
2.3. Summary of argument flow. Notation: With some abuse of notation, we will denote by $\left\{a_{n}\right\}$ the sequence $\left(a_{n}\right)_{n \in \mathbb{N}}$, and write $\left\{a_{n_{l}}\right\},\left\{a_{n_{l_{r}}}\right\}$ for subsequences $\left(a_{n_{l}}\right)_{l \in \mathbb{N}},\left(a_{n_{l_{r}}}\right)_{r \in \mathbb{N}}$, respectively. By $F$ we will refer to the functional $F_{\mathbf{q}, \boldsymbol{\lambda}}(x)$ in (2.1). We demonstrate that for our set of iterates $\left\{x^{n}\right\}$ from (1.6), we have convergence to the minimizing value, i.e. $\lim _{n \rightarrow \infty} F\left(x^{n}\right)=F(\bar{x})$, where $\bar{x}$ is such that $F(\bar{x}) \leq F(x)$ for all $x$. Under some conditions on $F$, the minimizer will be unique. In that case, we have that $x^{n} \rightarrow \bar{x}$. These statements will all follow from a few properties of $F$ and $G$ (from (2.1) and (2.6)) and the sequence of iterates $x^{n}$ from (1.6), which we now state, and which will be proved in Section 3:

(1) $0 \leq F\left(x^{n}\right) \leq G\left(x^{n}, x^{n}, w^{n}, \epsilon_{n}\right), \forall n$.

(2) $G\left(x^{n}, x^{n}, w^{n}, \epsilon_{n}\right) \leq G\left(x^{n-1}, x^{n-1}, w^{n-1}, \epsilon_{n-1}\right), \forall n$.

(3) $\exists$ subsequence $\left\{x^{n_{l}}\right\}$ of $\left\{x^{n}\right\}$ for which $\lim _{l \rightarrow \infty}\left[G\left(x^{n_{l}}, x^{n_{l}}, w^{n_{l}}, \epsilon_{n_{l}}\right)-F\left(x^{n_{l}}\right)\right]=0$.

(4) $\left\|x^{n}\right\|$ is bounded, which implies that any subsequence of $\left\{x^{n}\right\}$ has a weakly convergent subsequence; in particular $\left\{x^{n_{l}}\right\}$ has a convergent subsequence $\left\{x^{n_{l_{r}}}\right\}$.

(5) The limit $\bar{x}$ of the particular convergent subsequence $\left\{x^{n_{l_{r}}}\right\}$ satisfies the optimality conditions of $F$ (i.e. $F(\bar{x}) \leq F(x)$ for all $x)$.

We now show that these statements suffice to conclude that $\lim _{n \rightarrow \infty} F\left(x^{n}\right)=F(\bar{x})$, an important result, as it states that the iterates converge to the minimizing value of the functional. First, let us define the sequence $\left\{g_{n}\right\}:=G\left(x^{n}, x^{n}, w^{n}, \epsilon_{n}\right)$. Note from (1) and (2) that $\left\{g_{n}\right\}$ is bounded from below and monotonically decreasing; it follows that this sequence converges as $n \rightarrow \infty$, say to some $\bar{g}$. Consequently, $\left\{G\left(x^{n_{l}}, x^{n_{l}}, w^{n_{l}}, \epsilon_{n_{l}}\right)\right\}=\left\{g_{n_{l}}\right\}$ converges to $\bar{g}$ as $l \rightarrow \infty$. By (3) it then follows that $\left\{F\left(x^{n_{l}}\right)\right\}$ also converges to $\bar{g}$ as $l \rightarrow \infty$. Since we know that $x^{n_{l_{r}}} \rightarrow \bar{x}$, it follows from the continuity of $F$ that $\left\{F\left(x^{n_{l_{r}}}\right)\right\} \rightarrow\{F(\bar{x})\}$; consequently $\bar{g}=F(\bar{x})$ and hence $F\left(x^{n_{l}}\right) \rightarrow F(\bar{x})$ as $l \rightarrow \infty$, where $F(\bar{x}) \leq F(x)$ for all $x$.

Finally, we like to show that $F\left(x^{n}\right) \rightarrow F(\bar{x})$. Note that for any $\sigma>0, \exists L$ such that $\forall l \geq L$ we have that $\left|F\left(x^{n_{l}}\right)-F(\bar{x})\right|<\sigma$. Next, for every $n \geq n_{l} \geq l$, we have that:

$$
F\left(x^{n_{l}}\right)=g_{n_{l}} \geq g_{n}=G\left(x^{n}, x^{n}, w^{n}, \epsilon_{n}\right) \geq F\left(x^{n}\right)
$$

where $g_{n_{l}} \geq g_{n}$ since $n_{l} \leq n$. So this means that $F\left(x^{n_{l}}\right) \geq F\left(x^{n}\right)$ and we know from before that $\left|F\left(x^{n_{l}}\right)-F(\bar{x})\right|=$ $F\left(x^{n_{l}}\right)-F(\bar{x})<\sigma$, which implies that $F\left(x^{n}\right)-F(\bar{x})<\sigma$ for $n \geq n_{l}$, where we have used that $F(\bar{x}) \leq F(x)$ for all $x$. It follows that $F\left(x^{n}\right) \rightarrow F(\bar{x})$. This implies, in particular, that for any accumulation point $\hat{x}$ of $\left\{x^{n}\right\}$, we have $F(\hat{x})=F(\bar{x})$ (since $\hat{x}$ is the limit of a subsequence of $\left\{x^{n}\right\}$ and $F$ is continuous). In the case that the minimizer of $F$ is unique and equal to $\bar{x}$, it follows that $\bar{x}$ is the only possible accumulation point of $\left\{x^{n}\right\}$, i.e. that $x^{n} \rightarrow \bar{x}$. The majority of the work in the convergence argument which follows goes into introducing a proper construction for the $\left\{\epsilon_{n}\right\}$ sequence and showing that the properties (1) - (5) hold for this choice.

\section{Analysis of the IRLS algorithm}

Having set out the fundamentals (derivation of the scheme and outline of the convergence proof), we now analyze the IRLS scheme in (1.6), with weights $w_{k}^{n}$ defined by (1.4) and $\left\{\epsilon_{n}\right\}$ as defined by (1.5); we establish convergence by proving properties (1) to (5) from Section 2.3. We will assume that $\|A\|_{2}<1$. (I.e., $A$ has spectral or operator norm, or equivalently largest singular value, less than 1 , which can be accomplished by simple rescaling. The largest singular value can typically be estimated accurately using a few iterations of the power scheme.)

Lemma 3.1. Let the surrogate functional $G$ be given by (2.6) of Lemma 2.2 and $F$ be the functional in (2.1). Then property (1) above holds.

Proof. The proof follows by direct verification using the result of Remark 2.3.

$$
G\left(x^{n}, x^{n}, w^{n}, \epsilon_{n}\right)=\left\|A x^{n}-b\right\|_{2}^{2}+2 \sum_{k=1}^{N} \lambda_{k}\left(\left(x_{k}^{n}\right)^{2}+\left(\epsilon_{n}\right)^{2}\right)^{\frac{q_{k}}{2}} \geq F\left(x^{n}\right)=\left\|A x^{n}-b\right\|_{2}^{2}+2 \sum_{k=1}^{N} \lambda_{k}\left|x_{k}^{n}\right|^{q_{k}} \geq 0 .
$$


Lemma 3.2. Assume that the spectral norm of $A$ is bounded by 1, i.e. $\|A\|_{2}<1$. Then the sequence of iterates $\left\{x^{n}\right\}$ generated by (1.6) satisfies $\left\|x^{n}-x^{n-1}\right\|_{2} \rightarrow 0$ and the $x_{n}$ are bounded in $\ell_{1}$-norm $\left(\left\|x^{n}\right\|_{1} \leq K\right.$ for some $K \in \mathbb{R})$.

Proof. Using the results from Lemma 2.2, we write down a sequence of inequalities:

$$
\begin{aligned}
G\left(x^{n+1}, x^{n+1}, w^{n+1}, \epsilon_{n+1}\right) & \leq G\left(x^{n+1}, x^{n+1}, w^{n}, \epsilon_{n+1}\right) \quad[A] \\
& \leq G\left(x^{n+1}, x^{n}, w^{n}, \epsilon_{n+1}\right) \quad[B] \\
& \leq G\left(x^{n+1}, x^{n}, w^{n}, \epsilon_{n}\right) \quad[C] \\
& \leq G\left(x^{n}, x^{n}, w^{n}, \epsilon_{n}\right) . \quad[D]
\end{aligned}
$$

We now offer explanations for $[A-D]$. First, $[A]$ follows from $w^{n+1}=\arg \min _{w} G\left(x^{n+1}, a, w, \epsilon_{n+1}\right)$. Next for $[B]$, we have:

$$
G\left(x^{n+1}, x^{n}, w^{n}, \epsilon_{n+1}\right)-G\left(x^{n+1}, x^{n+1}, w^{n}, \epsilon_{n+1}\right)=\left\|x^{n}-x^{n+1}\right\|_{2}^{2}-\left\|A\left(x^{n}-x^{n+1}\right)\right\|_{2}^{2},
$$

Now $\left\|A\left(x-x^{n}\right)\right\|_{2} \leq\|A\|_{2}\left\|x-x^{n}\right\|_{2}<\left\|x-x^{n}\right\|_{2}$ for $\|A\|_{2}<1$, so that $\left\|x-x^{n}\right\|_{2}^{2}-\left\|A\left(x-x^{n}\right)\right\|_{2}^{2}>0$. Next, $[C]$ follows from $\epsilon_{n+1} \leq \epsilon_{n}$ (directly from (1.5)). Finally, [D] follows from $x^{n+1}=\arg \min _{x} G\left(x, x^{n}, w^{n}, \epsilon_{n}\right)$.

We now set up a telescoping sum of non-negative terms, using the inequalities $[A-D]$ above:

$$
\begin{aligned}
& \sum_{n=1}^{P}\left(G\left(x^{n+1}, x^{n}, w^{n}, \epsilon_{n+1}\right)-G\left(x^{n+1}, x^{n+1}, w^{n}, \epsilon_{n+1}\right)\right) \\
& \quad \leq \sum_{n=1}^{P}\left(G\left(x^{n}, x^{n}, w^{n}, \epsilon_{n}\right)-G\left(x^{n+1}, x^{n+1}, w^{n+1}, \epsilon_{n+1}\right)\right) \\
& \quad=G\left(x^{1}, x^{1}, w^{1}, \epsilon_{1}\right)-G\left(x^{P+1}, x^{P+1}, w^{P+1}, \epsilon_{P+1}\right) \leq G\left(x^{1}, x^{1}, w^{1}, \epsilon_{1}\right)=: C \in \mathbb{R},
\end{aligned}
$$

where we have used that $G\left(x^{n}, x^{n}, w^{n}, \epsilon_{n}\right)$ is always $\geq 0$. Using (3.1), it follows that:

$$
\sum_{n=1}^{P}\left(\left\|x^{n}-x^{n+1}\right\|_{2}^{2}-\left\|A\left(x^{n}-x^{n+1}\right)\right\|_{2}^{2}\right) \leq C .
$$

Since $\left\|A\left(x^{n}-x^{n+1}\right)\right\|_{2}^{2} \leq\|A\|_{2}^{2}\left\|x^{n}-x^{n+1}\right\|_{2}^{2}$ and $\|A\|_{2}<1$ :

$$
\begin{aligned}
\left\|x^{n}-x^{n+1}\right\|_{2}^{2}-\left\|A\left(x^{n}-x^{n+1}\right)\right\|_{2}^{2} & \geq\left\|x^{n}-x^{n+1}\right\|_{2}^{2}-\|A\|_{2}^{2}\left\|x^{n}-x^{n+1}\right\|_{2}^{2} \\
& =\gamma\left\|x^{n}-x^{n+1}\right\|_{2}^{2},
\end{aligned}
$$

where $\gamma:=\left(1-\|A\|_{2}^{2}\right)>0$. Consequently, we have:

$$
\begin{array}{cl}
\gamma \sum_{n=1}^{P}\left\|x^{n}-x^{n+1}\right\|_{2}^{2} & \leq \sum_{n=1}^{P}\left(\left\|x^{n}-x^{n+1}\right\|_{2}^{2}-\left\|A\left(x^{n}-x^{n+1}\right)\right\|_{2}^{2}\right) \leq C \\
\Longrightarrow & \sum_{n=1}^{\infty}\left\|x^{n}-x^{n+1}\right\|_{2}^{2}<\infty \\
\Longrightarrow & \left\|x^{n}-x^{n+1}\right\|_{2} \rightarrow 0 .
\end{array}
$$

To prove that the $\left\{x^{n}\right\}$ are bounded, we use the result from Remark 2.3:

$$
G\left(x^{n}, x^{n}, w^{n}, \epsilon_{n}\right)=\left\|A x^{n}-b\right\|_{2}^{2}+2 \sum_{k=1}^{N} \lambda_{k}\left(\left(x_{k}^{n}\right)^{2}+\left(\epsilon_{n}\right)^{2}\right)^{\frac{q_{k}}{2}} \geq \lambda_{k}\left|x_{k}^{n}\right|^{q_{k}},
$$


It follows that:

$$
\left|x_{k}^{n}\right| \leq\left(\frac{1}{\lambda_{k}} G\left(x^{n}, x^{n}, w^{n}, \epsilon_{n}\right)\right)^{\frac{1}{q_{k}}} \leq\left(\frac{1}{\lambda_{k}} G\left(x^{1}, x^{1}, w^{1}, \epsilon_{1}\right)\right)^{\frac{1}{q_{k}}} \leq \max _{k \in\{1, \ldots, N\}}\left(\frac{1}{\lambda_{k}} G\left(x^{1}, x^{1}, w^{1}, \epsilon_{1}\right)\right)^{\frac{1}{q_{k}}}=: C_{1}
$$

This implies the boundedness of $\left\{x^{n}\right\}$, since $\left\|x^{n}\right\|_{1}=\sum_{k=1}^{N}\left|x_{k}^{n}\right| \leq N C_{1}$.

By Lemma 3.2 we have that property (2) holds; moreover (4) (the boundedness of the $\left\|x^{n}\right\|_{1}$ ) is established as well. The next lemma demonstrates property (3) and the existence of a convergent subsequence $x^{n_{l_{r}}}$.

Lemma 3.3. There exists a subsequence $\left\{\epsilon_{n_{l}}\right\}$ of $\left\{\epsilon_{n}\right\}$ such that every member of the subsequence is defined by:

$$
\epsilon_{n_{l}}=\left(\left\|x^{n_{l}}-x^{n_{l}-1}\right\|_{2}+\alpha^{n_{l}}\right)^{\frac{1}{2}}<\epsilon_{n_{l}-1} .
$$

Additionally, there is a subsequence $\left\{n_{l_{r}}\right\}$ of this subsequence such that $\left\{x^{n_{l_{r}}}\right\}_{r}$ is convergent.

Proof. By the definition of the $\epsilon_{n}$ 's in (1.5) and by Lemma 3.2, we know that $\epsilon_{n} \rightarrow 0$, since $\left\|x^{n}-x^{n-1}\right\| \rightarrow 0$ and $\alpha^{n} \rightarrow 0$. It follows that a subsequence $\left\{n_{l}\right\}$ must exist such that $\epsilon_{n_{l}}<\epsilon_{n_{l}-1}$, for otherwise, the monotonicity $\epsilon_{n+1} \leq \epsilon_{n}$ combined with $\epsilon_{n}>0$ for all $n$ would imply the existence of $N_{0}$ such that for $n \geq N_{0}, \epsilon_{n+1}=\epsilon_{n}$, implying that the sequence of $\epsilon_{n}$ 's would not converge to zero. The fact that $n_{l_{r}}$ exists is a consequence of the boundedness of the iterates $\left\{x^{n}\right\}$ and hence that of $\left\{x^{n_{l}}\right\}$, Lemma 3.2, and the standard fact that any bounded sequence in $\mathbb{R}^{N}$ has at least one accumulation point.

By Lemma 3.3 and Lemma 3.2, we have that $\epsilon_{n_{l}} \rightarrow 0$ as $l \rightarrow \infty$. Thus, together with (2.12), it follows that (3) holds.

Lemma 3.4. The limit $\bar{x}$ of the converging subsequence $\left\{x^{n_{l_{r}}}\right\}$ satisfies the optimality conditions (2.2) of the convex functional (2.1):

$$
\begin{aligned}
& \left\{A^{T}(b-A x)\right\}_{k}=\lambda_{k} \operatorname{sgn}\left(x_{k}\right) q_{k}\left|x_{k}\right|^{q_{k}-1}, \quad x_{k} \neq 0 \quad\left(1 \leq q_{k} \leq 2\right) \\
& \left|\left\{A^{T}(b-A x)\right\}_{k}\right| \leq \lambda_{k}, \quad x_{k}=0 \quad\left(q_{k}=1\right) \\
& \left\{A^{T}(b-A x)\right\}_{k}=0, \quad x_{k}=0 \quad\left(q_{k}>1\right)
\end{aligned}
$$

Proof. For each $k$, we consider three separate cases, depending on the limit $\bar{x}_{k}$.

(1) $\bar{x}_{k} \neq 0$ and $1 \leq q_{k} \leq 2$,

(2) $\bar{x}_{k}=0$ and $q_{k}=1$,

(3) $\bar{x}_{k}=0$ and $q_{k}>1$.

Since $x^{n_{l_{r}}} \rightarrow \bar{x}$, and since $\left\|x^{n}-x^{n+1}\right\| \rightarrow 0$ (by Lemma 3.2), we have that: $x_{k}^{n_{l_{r}}+1} \rightarrow \bar{x}_{k}$. We can rewrite the iterative scheme (1.6) as:

$$
x_{k}^{n+1}\left(1+\lambda_{k} q_{k} w_{k}^{n}\right)=x_{k}^{n}+\left\{A^{T}\left(b-A x^{n}\right)\right\}_{k}
$$

Specializing this to $\left\{x^{n_{l_{r}}}\right\}$ and reordering terms, we have:

$$
\lambda_{k} q_{k} w_{k}^{n_{l_{r}}} x_{k}^{n_{l_{r}}+1}=x_{k}^{n_{l_{r}}}-x_{k}^{n_{l_{r}}+1}+\left\{A^{T}\left(b-A x^{n_{l_{r}}}\right)\right\}_{k} .
$$

Since the right hand side converges to a limit as $r \rightarrow \infty$, so must the left hand side; we obtain:

$$
\lim _{r \rightarrow \infty} w_{k}^{n_{l_{r}}} x_{k}^{n_{l_{r}+1}}=\frac{1}{\lambda_{k} q_{k}}\left\{A^{T}(b-A \bar{x})\right\}_{k} .
$$

We will use this to compute $\left\{A^{T}(b-A \bar{x})\right\}_{k}$ and to verify that (2.2) is satisfied. We are thus interested in the value of $\lim _{r \rightarrow \infty} w_{k}^{n_{l_{r}}} x_{k}^{n_{l_{r}}+1}$. 
In case (1), $\lim _{r \rightarrow \infty} x_{k}^{n_{l_{r}}}=\overline{x_{k}} \neq 0$, we obtain

$$
\lim _{r \rightarrow \infty} w_{k}^{n_{l_{r}}} x_{k}^{n_{l_{r}}+1}=\lim _{l \rightarrow \infty} w_{k}^{n_{l_{r}}} x_{k}^{n_{l_{r}}} \frac{x_{k}^{n_{l_{r}}+1}}{x_{k}^{n_{l_{r}}}}=\lim _{l \rightarrow \infty} x_{k}^{n_{l_{r}}} w_{k}^{n_{l_{r}}}
$$

where we have used that $\lim _{r \rightarrow \infty} \frac{x_{k}^{n_{l_{r}}+1}}{x_{k}^{n_{l_{r}}}}=1$, since $\left\|x^{n+1}-x^{n}\right\| \rightarrow 0$. Using (1.4), it follows that:

$$
\begin{aligned}
\lim _{r \rightarrow \infty} w_{k}^{n_{l_{r}}} x_{k}^{n_{l_{r}}+1} & =\lim _{r \rightarrow \infty} \frac{x_{k}^{n_{l_{r}}}}{\left[\left(x_{k}^{n_{l_{r}}}\right)^{2}+\left(\epsilon_{n_{l_{r}}}\right)^{2}\right]^{\frac{2-q_{k}}{2}}}=\frac{\overline{x_{k}}}{\left(\left(\overline{x_{k}}\right)^{2}+0\right)^{\frac{2-q_{k}}{2}}} \\
& =\frac{\operatorname{sgn}\left(\overline{x_{k}}\right)\left|\overline{x_{k}}\right|}{\left|\overline{x_{k}}\right|^{2-q_{k}}}=\operatorname{sgn}\left(\overline{x_{k}}\right)\left|\overline{x_{k}}\right|^{q_{k}-1} .
\end{aligned}
$$

Thus, from (3.3), we obtain that: $\left\{A^{T}(b-A \bar{x})\right\}_{k}=\lambda_{k} q_{k} \operatorname{sgn}\left(\overline{x_{k}}\right)\left|x_{k}\right|^{q_{k}-1}$, in accordance with (2.2).

In case (2) and (3), $\lim _{r \rightarrow \infty} x^{n_{l_{r}}}=\overline{x_{k}}=0$, and we still have that (3.3) holds. Writing out (1.6) for $x^{n_{k_{l_{r}}}}$ in terms of $x^{n_{k_{l_{r}}}-1}$, we obtain:

$$
\lambda_{k} q_{k} w_{k}^{n_{l_{r}}-1} x_{k}^{n_{l_{r}}}=x_{k}^{n_{l_{r}}-1}-x_{k}^{n_{l_{r}}}+\left\{A^{T}\left(b-A x^{n_{l_{r}}-1}\right)\right\}_{k} .
$$

which gives the limit:

$$
\lim _{r \rightarrow \infty} w_{k}^{n_{l_{r}}-1} x_{k}^{n_{l_{r}}}=\frac{1}{\lambda_{k} q_{k}}\left\{A^{T}(b-A \bar{x})\right\}_{k} .
$$

We define $\beta_{k}$ to be:

$$
\beta_{k}:=\frac{1}{\lambda_{k} q_{k}}\left\{A^{T}(b-A \bar{x})\right\}_{k}
$$

To prove that (2.2) is satisfied, we must show that $\left|\beta_{k}\right| \leq 1$ for case (2) and that $\beta_{k}=0$ for case (3).

We first write down some relations involving $\beta_{k}$ which we will use. Note that by (3.4), $\lim _{r \rightarrow \infty} w_{k}^{n_{l_{r}}-1} x_{k}^{n_{l_{r}}}=$ $\beta_{k}$. If $\beta_{k} \neq 0$, it follows that for every $\sigma \in(0,1), \exists r_{0}$ such that for every $r \geq r_{0}$ :

$$
\left(w_{k}^{n_{l_{r}}-1} x_{k}^{n_{l_{r}}}\right)^{2}>(1-\sigma) \beta_{k}^{2} \Longrightarrow\left(x_{k}^{n_{l_{r}}}\right)^{2}>(1-\sigma) \beta_{k}^{2}\left(w_{k}^{n_{l_{r}}-1}\right)^{-2}=(1-\sigma) \beta_{k}^{2}\left(\left(x_{k}^{n_{l_{r}}-1}\right)^{2}+\left(\epsilon_{n_{l_{r}}-1}\right)^{2}\right)^{2-q_{k}}
$$

Since $\epsilon_{n_{l_{r}}}<\epsilon_{n_{l_{r}}-1}$, it follows that for $r$ sufficiently large::

$$
\begin{aligned}
\left(x_{k}^{n_{l_{r}}}\right)^{2} & >(1-\sigma) \beta_{k}^{2}\left(\left(x_{k}^{n_{l_{r}}-1}\right)^{2}+\left(\epsilon_{n_{l_{r}}}\right)^{2}\right)^{2-q_{k}} \\
& =(1-\sigma) \beta_{k}^{2}\left(\left(x_{k}^{n_{l_{r}}-1}\right)^{2}+\left\|x^{n_{l_{r}}}-x^{n_{l_{r}}-1}\right\|_{2}+\alpha^{n_{l_{r}}}\right)^{2-q_{k}} \\
& \geq(1-\sigma) \beta_{k}^{2}\left(\left(x_{k}^{n_{l_{r}}-1}\right)^{2}+\left|x_{k}^{n_{l_{r}}}-x_{k}^{n_{l_{r}}-1}\right|+\alpha^{n_{l_{r}}}\right)^{2-q_{k}} \\
& >(1-\sigma) \beta_{k}^{2}\left(\left(x_{k}^{n_{l_{r}}-1}\right)^{2}+\left|x_{k}^{n_{l_{r}}}-x_{k}^{n_{l_{r}}-1}\right|\right)^{2-q_{k}}
\end{aligned}
$$

where we have used in the last part that $\alpha^{n_{l_{r}}} \rightarrow 0$. To simplify notation, let us set $u=x_{k}^{n_{l_{r}}-1}$ and $v=$ $x_{k}^{n_{l_{r}}}-x_{k}^{n_{l_{r}}-1}$. Then in terms of $u$ and $v$, we have:

$$
(u+v)^{2}>(1-\sigma) \beta_{k}^{2}\left(u^{2}+|v|\right)^{2-q_{k}}
$$

Notice that for any $K>0$ :

$$
0 \leq\left(\sqrt{K} u-\frac{1}{\sqrt{K}} v\right)^{2}=K u^{2}+\frac{1}{K} v^{2}-2 u v
$$


It follows that:

$$
(u+v)^{2}=u^{2}+2 u v+v^{2} \leq u^{2}+K u^{2}+\frac{1}{K} v^{2}+v^{2}=(1+K) u^{2}+\left(1+\frac{1}{K}\right) v^{2}
$$

Using (3.6) in (3.5), we get:

$$
(1-\sigma) \beta_{k}^{2}\left(u^{2}+|v|\right)^{2-q_{k}}<(1+K) u^{2}+\left(1+\frac{1}{K}\right) v^{2}
$$

Let us now consider case (2) where $q_{k}=1$. We assume that $\beta_{k}>1$ and derive a contradiction. Rearranging terms in (3.7) yields:

$$
\left((1-\sigma) \beta_{k}^{2}-(1+K)\right) u^{2}<\left(1+\frac{1}{K}\right) v^{2}-(1-\sigma) \beta_{k}^{2}|v|=\left(\left(1+\frac{1}{K}\right)|v|-(1-\sigma) \beta_{k}^{2}\right)|v|
$$

Since we assume that $\left|\beta_{k}^{2}\right|>1$, we can choose our $\sigma<1$ small enough such that $(1-\sigma) \beta_{k}^{2}>1$; once $\sigma$ is fixed, we can choose $K>0$ small enough such that $(1-\sigma) \beta_{k}^{2} \geq(1+K)$; with these choices of $\sigma$ and $K$, the left hand side of $(3.8) \geq 0$. With this fixed choice of $\sigma$ and $K$ we analyze the right hand side of (3.8). Note that by Lemma 3.2, we have that $\left\|x^{n_{l_{r}}}-x^{n_{l_{r}}-1}\right\|_{2} \rightarrow 0$ as $r \rightarrow \infty$. This means that $|v| \rightarrow 0$ as $r \rightarrow \infty$. For sufficiently large $r$, we will have $|v|<\left(1+\frac{1}{K}\right)^{-1}(1-\sigma) \beta_{k}^{2}$, implying that the right hand side of (3.8) would then be $\leq 0$. This is in contradiction with the left hand side of this strict inequality (3.8) being $\geq 0$. It follows that the assumption $\left|\beta_{k}\right|>1$ is not correct. Hence, we have $\left|\beta_{k}\right| \leq 1$ which implies that $\left|\left\{A^{T}(b-A \bar{x})\right\}_{k}\right| \leq \lambda_{k}$, consistent with (2.2).

Finally, consider case (3) with $q_{k}>1$. We assume that $\left|\beta_{k}\right|>0$ and derive a contradiction. In this case, (3.7) does not simplify further:

$$
\beta_{k}^{2}(1-\sigma)\left(u^{2}+|v|\right)^{2-q_{k}}<(1+K) u^{2}+\left(1+\frac{1}{K}\right) v^{2} \quad \text { for all } K>0 .
$$

This means in particular that:

$$
\begin{aligned}
& \beta_{k}^{2}(1-\sigma) u^{2\left(2-q_{k}\right)}<(1+K) u^{2}+\left(1+\frac{1}{K}\right) v^{2} \text { and } \\
& \beta_{k}^{2}(1-\sigma)|v|^{\left(2-q_{k}\right)}<(1+K) u^{2}+\left(1+\frac{1}{K}\right) v^{2} .
\end{aligned}
$$

Then the average of the terms is also smaller than this quantity:

$$
\frac{1}{2} \beta_{k}^{2}(1-\sigma)\left(u^{2\left(2-q_{k}\right)}+|v|^{\left(2-q_{k}\right)}\right)<(1+K) u^{2}+\left(1+\frac{1}{K}\right) v^{2} .
$$

Rearranging terms again, we have:

$$
u^{2\left(2-q_{k}\right)}\left(\frac{1}{2} \beta_{k}^{2}(1-\sigma)-(1+K) u^{2\left(q_{k}-1\right)}\right)<\left(1+\frac{1}{K}\right) v^{2}-\frac{1}{2} \beta_{k}^{2}(1-\sigma)|v|^{\left(2-q_{k}\right)} .
$$

Since $q_{k}>1$ and thus $2-q_{k}<1$, we have that for $v$ sufficiently small (obtained by taking $r$ sufficiently large), the right hand side is negative, by the same logic as in the previous case (because $\left|\beta_{k}\right|>0$ by assumption, the first term will go to zero faster than the second when $v \rightarrow 0$ as $r \rightarrow \infty)$. Thus, by the above inequality, for $r$ sufficiently large, the left hand side, bounded above by the negative right hand side, must be negative as well. Since $u^{2\left(2-q_{k}\right)}$ is non-negative, that is possible only when:

$$
\frac{1}{2} \beta_{k}^{2}(1-\sigma)-(1+K) u^{2\left(q_{k}-1\right)}<0
$$


for $r$ sufficiently large. However, since $\lim _{r \rightarrow \infty} u^{2\left(q_{k}-1\right)}=\lim _{r \rightarrow \infty}\left(x_{k}^{n_{l_{r}}-1}\right)^{2\left(q_{k}-1\right)}=0$, this condition cannot be satisfied for large $r$. This contradicts our original assumption that $\left|\beta_{k}\right|>0$. Hence, we conclude that $\beta_{k}=0$. It follows that $\left\{A^{T}(b-A \bar{x})\right\}_{k}=0$, which is the right optimality condition.

Lemma 3.4, together with the proceeding Lemmas in this section, show that properties (1) to (5) of Section 2.3 hold. It thus follows from the argument in Section 2.3 that we have $F\left(x^{n}\right) \rightarrow F(\bar{x})$.

\section{Numerics}

We now discuss some aspects of the numerical implementation and performance of the IRLS algorithm. We first illustrate performance for the case $q_{k}=1$ for all $k$, where it's easiest to compare with existing algorithms. Then we discuss a simple example concerning a case where different values of $q_{k}$ can be used. An implementation of the scheme as given by (1.6) has the same computational complexity as ISTA in (1.2). Not surprisingly, the performance of the two schemes is also similar. However, our numerical experiments indicate that the speed-up idea behind FISTA as described in [1] is also effective for the IRLS algorithm. FISTA was designed to minimize the function $f(x)+g(x)$, where $f$ is a continuously differentiable convex function with Lipschitz continuous gradient (i.e., $\|\nabla f(x)-\nabla f(y)\|_{2} \leq L\|x-y\|_{2}$ for some constant $L>0$ ), and $g$ is a continuous convex function such as $2 \tau\|x\|_{1}$ in the $\ell_{1}$-penalized functional. FISTA uses the proximal mapping function:

$$
p_{L}(y)=\arg \min _{x}\left\{g(x)+\frac{L}{2}\left\|x-\left(y-\frac{1}{L} \nabla f(y)\right)\right\|_{2}^{2}\right\}
$$

to define the following algorithm:

$$
\begin{aligned}
y^{1} & =x^{0} \in \mathbb{R}^{N} \quad, \quad t_{1}=1 \quad, \quad \text { and for } n=1,2, \ldots, \\
x^{n+1} & =p_{L}\left(y^{n}\right)=\arg \min _{x}\left\{g(x)+\frac{L}{2}\left\|x-\left(y^{n}-\frac{1}{L} \nabla f\left(y^{n}\right)\right)\right\|^{2}\right\} \\
t_{n+1} & =\frac{1+\sqrt{1+4 t_{n}^{2}}}{2} \\
y^{n+1} & =x^{n+1}+\frac{t_{n}-1}{t_{n+1}}\left(x^{n+1}-x^{n}\right) .
\end{aligned}
$$

In the case that $f(x)=\|A x-b\|_{2}^{2}$ and $g(x)=2 \tau\|x\|_{1}$, we obtain:

$$
\begin{aligned}
\|\nabla f(x)-\nabla f(y)\|_{2} & =\left\|2 A^{T} A x-2 A^{T} A y\right\|_{2}=\left\|2 A^{T} A(x-y)\right\|_{2} \\
& \leq 2\left\|A^{T} A\right\|_{2}\|x-y\|_{2},
\end{aligned}
$$

which implies that when $A$ is scaled such that $\|A\|_{2} \approx 1$, the Lipschitz constant can be taken to be $L=2$. It follows that:

$$
g(x)+\frac{L}{2}\left\|x-\left(y-\frac{1}{L} \nabla f(y)\right)\right\|_{2}^{2}=2 \tau\|x\|_{1}+\left\|x-\left(y-A^{T}(A y-b)\right)\right\|_{2}^{2} .
$$

Using (4.2) in (4.1), we obtain:

$$
x^{n+1}=\arg \min _{x}\left\{2 \tau\|x\|_{1}+\left\|x-\left(y^{n}-A^{T}\left(A y^{n}-b\right)\right)\right\|_{2}^{2}\right\}=\mathbb{S}_{\tau}\left(y^{n}-A^{T} A y^{n}+A^{T} b\right),
$$

where we have used (1.1). We note that (4.3) is very similar to the ISTA scheme in (1.2), except the thresholding is applied to $\left\{y^{n}\right\}$. In the same way, we can coin the FIRLS algorithm by performing the steps in (4.1), using

$$
x^{n+1}=\frac{1}{1+\tau\left[\left(y_{k}^{n}\right)^{2}+\left(\epsilon_{n}\right)^{2}\right]^{\frac{1}{2}}}\left\{y^{n}-A^{T} A y^{n}+A^{T} b\right\}_{k} \quad \text { for } \quad k=1, \ldots, N
$$


in place of (4.3). With the more general weights given by (1.4), we can specialize this algorithm to our functional (2.1).

We now demonstrate some results of simple numerical experiments. We begin with the $q_{k}=1$ case for all $k$. We also let the regularization parameter be the same for all $k$, setting $\lambda_{k}=\tau$. For the first test, we use two differently conditioned random matrices (built up via a reverse SVD procedure with orthogonal random matrices $U$ and $V$, obtained by performing a QR factorization on the Gaussian random matrices, and a custom diagonal matrix of singular values $S$, to form a $1000 \times 1000$ matrix $A=U S V^{T}$ ), and a sparse signal $x$ with $5 \%$ non-zeros. We form $b=A x$ and use the different algorithms to recover $\tilde{x}$ using a single run of 300 iterations with $\tau=\frac{\max \left|A^{T} b\right|}{10^{5}}$. In Figure 1, we plot the decrease of $\ell_{1}$-functional values $F_{1}\left(x^{n}\right)$ and recovery percent errors $100 \frac{\left\|x^{n}-x\right\|}{\|x\|}$ versus the iterate number $n$, using four algorithms: IRLS, FIRLS, ISTA, and FISTA for two matrix types: $A_{1}$, with singular values logspaced between 1 and 0.1 and $A_{2}$, with singular values logspaced between 1 and $10^{-4}$. We see that the performance of ISTA/IRLS and FISTA/FIRLS are mostly similar, with better recovery using FISTA in the well-conditioned case, but almost identical performance in the worst-conditioned case.

In Figure 2, we run a compressive sensing experiment. We again take the $1000 \times 1000$ matrix of type $A_{2}$. Now we use a staircase-like sparse vector $x$ with about $12 \%$ non-zeros. After we form $b=A x$, we zero out all but the first $\frac{1}{3}$ of the rows of $A$ and $b$ forming $A_{p}$ and $b_{p}$ (i.e. we only keep a portion of the measurements). We then recover solution $\tilde{x}$ using $A_{p}$ and $b_{p}$ while employing a continuation scheme across 20 different values of $\tau$, starting with a zero initial guess at $\tau=\max \left|A^{T} b\right|$ and proceeding down to $\tau=\frac{\max \left|A^{T} b\right|}{50000}$, while reusing the previous solutions as the initial guess at each new value of $\tau$. From Figure 2, we can see that the recovered solutions with FIRLS and FISTA are very similar.

We illustrate the use of the more general functional in (2.1) in Figure 3. We use the same setup as before, with the different algorithms running across multiple values of the regularization parameter $\tau$, which is fixed for all $k$. However, we use a more complicated input signal, whose first half is sparse and whose second half is entirely dense. For this reason, in the IRLS schemes, we take $q_{k}=1$ for the first half of the weights (for indices $k$ from 1 to $\frac{n}{2}$ ) and $q_{k}=1.9$ for the second half (for indices $k$ from $\frac{n}{2}+1$ to $n$ ). We observe that the recovered signal with the IRLS algorithms is superior to that of the ISTA/FISTA schemes which utilize $q_{k}=1$ for all entries. Of course, setting the values of $q_{k}$ for individual coefficients maybe difficult in practice unless one knows the distribution of the sparser and denser parts in advance, although in applications, some information of this nature may be available from the setup of the problem.

Finally, in Figure 4, we show the result of an image reconstruction experiment. We use two images, blurred with a Gaussian source and corrupted by Gaussian noise. The first image is $170 \times 120$ and the second is $125 \times 125$. In both cases, the blurring source is a $2 \mathrm{D}$ Gaussian function with support on a $9 \times 9$ grid with $\sigma=2.5$ and max amplitude of 2.9. We then take the blurred image (obtained via convolution with the blurring source) and add white Gaussian noise, so that the signal to noise ratio is 25 . We then recover a corrected image using an application of wavelet denoising followed by IRLS, from the blurred and noisy image. The IRLS algorithms is run over 30 parameters $\tau$ with 40 iterations each, in a setup similar to that used for Figure 2. The matrices we use in the inversion are derived from the blur source itself, so this is a non-blind deconvolution. The problem, however, is still challenging and the resulting images are much improved from their blurred and noisy counterparts. We have noticed that the use of $q_{k}<1$ can yield, in some instances, slighter sharper reconstructions in the same number of iterations. 

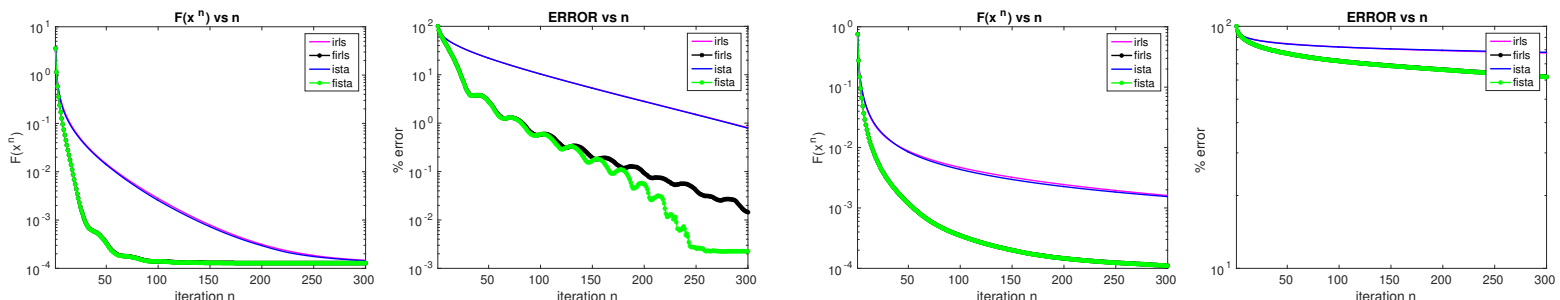

FiguRE 1. Functional values $F\left(x^{n}\right)$ and recovery percent errors $100 \frac{\left\|x^{n}-x\right\|}{\|x\|}$ versus the iterate number $n$ for better and worse conditioned matrices (medians over 10 trials).

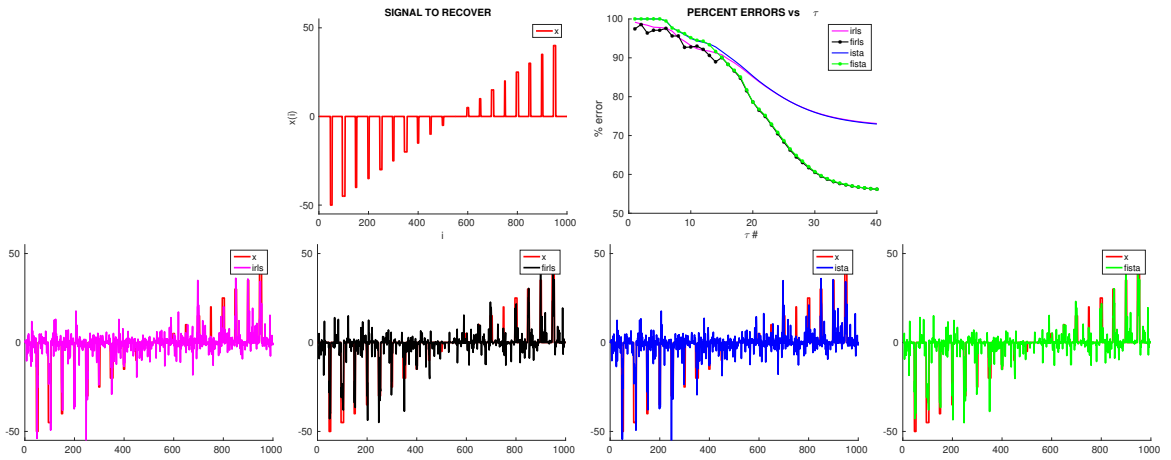

FiguRE 2. Row 1: sparse model $x$ and the recovery percent errors vs $\tau$. Row 2: final recovered solution with algorithms IRLS, FIRLS, ISTA, FISTA vs $x$.

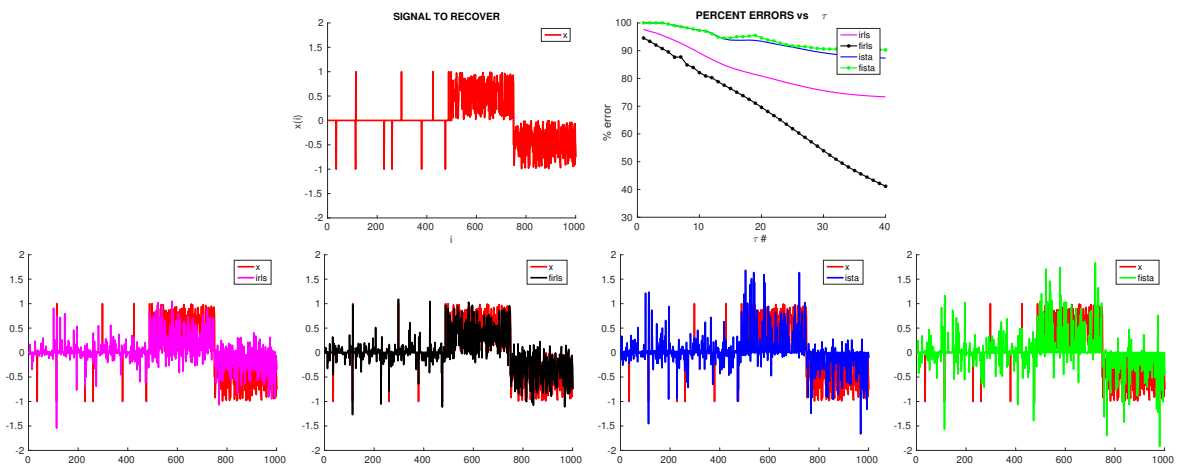

FiguRE 3. Row 1: half sparse / half dense model $x$ and the recovery percent errors vs $\tau$. Row 2: final recovered solution with algorithms IRLS, FIRLS, ISTA, FISTA vs $x$. 


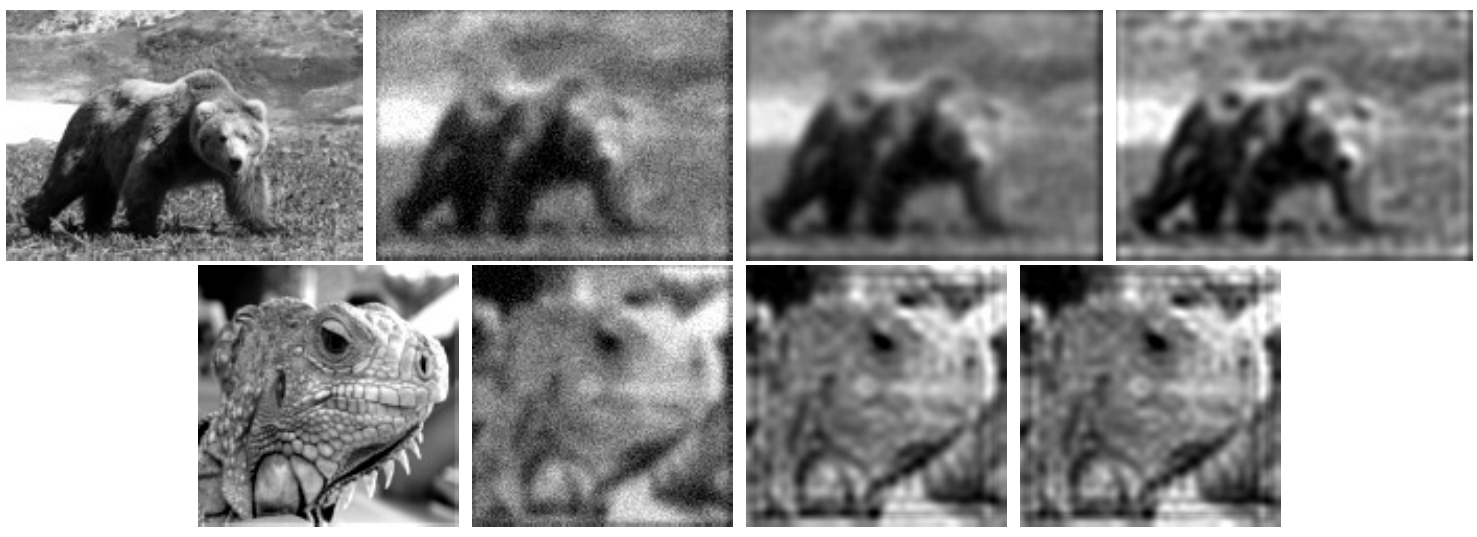

FIGURE 4. Two examples of blurred and noisy image reconstruction with IRLS. In each row from left to right: original image, blurred and noisy image, and two reconstructions with IRLS using $q=1$ and $q=0.85$.

\section{Conclusions}

This manuscript presents a new iterative algorithm for obtaining regularized solutions to least squares systems of equations with sparsity constraints. The proposed iteratively reweighted least squares algorithm extends the work of [5] and is similar in form to the popular ISTA and FISTA algorithms [4, 1]; it has the added benefit of being able to minimize a more general sparsity promoting functional. The main contribution of this work is the analysis of the algorithm, relying on matching the approximation rate to the original functional of a smoothened surrogate functional to the speed of convergence of the iterates; this methodology can likely also be applied to other situations. The presented IRLS algorithm (1.6) is very simple to implement and use; it offers performance similar to popular thresholding schemes, including the speedup benefit from the FISTA formulation. Because the surrogate functionals are all quadratic in the $x_{k}$, they lend themselves naturally to the use of a conjugate gradient approach, which enables further speed-up, as shown elsewhere [11, 7].

\section{References}

[1] Amir Beck and Marc Teboulle. A fast iterative shrinkage-thresholding algorithm for linear inverse problems. SIAM J. Imaging Sci., 2(1):183-202, 2009. 2, 13, 16

[2] Jian-Feng Cai, Stanley Osher, and Zuowei Shen. Linearized bregman iterations for compressed sensing. Mathematics of Computation, 78(267):1515-1536, 2009. 1

[3] Emmanuel J Candès and Michael B Wakin. An introduction to compressive sampling. Signal Processing Magazine, IEEE, 25(2):21-30, 2008. 1

[4] I. Daubechies, M. Defrise, and C. De Mol. An iterative thresholding algorithm for linear inverse problems with a sparsity constraint. Comm. Pure Appl. Math., 57(11):1413-1457, 2004. 1, 2, 16

[5] I. Daubechies, R. DeVore, M. Fornasier, and C. Sinan Güntürk. Iteratively reweighted least squares minimization for sparse recovery. Communications on Pure and Applied Mathematics, 63(1):1-38, 2010. 2, 16

[6] Mário AT Figueiredo, José M Bioucas-Dias, and Robert D Nowak. Majorization-minimization algorithms for wavelet-based image restoration. IEEE Transactions on Image processing, 16(12):2980-2991, 2007. 1, 2

[7] M. Fornasier, S. Peter, H. Rauhut, and S. Worm. Conjugate gradient acceleration of iteratively re-weighted least squares methods. ArXiv e-prints, September 2015. 3, 16

[8] Jean Jacques Fuchs. Convergence of a sparse representations algorithm applicable to real or complex data. IEEE Journal of Selected Topics in Signal Processing, 1(4):598-605, 2007. 2 
[9] Per Christian Hansen. The L-curve and its use in the numerical treatment of inverse problems. IMM, Department of Mathematical Modelling, Technical Universityof Denmark, 1999. 5

[10] Frederik J Simons, Ignace Loris, Guust Nolet, Ingrid C Daubechies, S Voronin, JS Judd, Ph A Vetter, J Charléty, and $\mathrm{C}$ Vonesch. Solving or resolving global tomographic models with spherical wavelets, and the scale and sparsity of seismic heterogeneity. Geophysical journal international, 187(2):969-988, 2011. 1, 2

[11] S. Voronin. Regularization of linear systems with sparsity constraints with applications to large scale inverse problems. PhD thesis, Princeton University, Nov 2012. 1, 3, 16

[12] S. Voronin and R. Chartrand. A new generalized thresholding algorithm for inverse problems with sparsity constraints. ICASSP, 2013. 2

[13] S. Voronin, G. Ozkaya, and D. Yoshida. Convolution based smooth approximations to the absolute value function with application to non-smooth regularization. ArXiv e-prints, August 2014. 2

[14] John Wright, Arvind Ganesh, Shankar Rao, Yigang Peng, and Yi Ma. Robust principal component analysis: Exact recovery of corrupted low-rank matrices via convex optimization. In Advances in neural information processing systems, pages 2080-2088, 2009. 1

[15] Allen Y Yang, S Shankar Sastry, Arvind Ganesh, and Yi Ma. Fast 11-minimization algorithms and an application in robust face recognition: A review. In Image Processing (ICIP), 2010 17th IEEE International Conference on, pages 1849-1852. IEEE, 2010. 1

[16] Junfeng Yang and Yin Zhang. Alternating direction algorithms for 11-problems in compressive sensing. SIAM journal on scientific computing, 33(1):250-278, 2011. 1, 2

[17] Wotao Yin, Stanley Osher, Donald Goldfarb, and Jerome Darbon. Bregman iterative algorithms for 11-minimization with applications to compressed sensing. SIAM Journal on Imaging Sciences, 1(1):143-168, 2008. 1

Mathematics, Tufts University, Medford, MA, 02155, USA.

Mathematics, Duke University, Durham, NC, 27708, USA. 